\title{
Spatial Intensity Correction of Fluorescent Confocal Laser Scanning Microscope Images
}

\author{
Sang-Chul Lee and Peter Bajcsy \\ National Center for Supercomputing Applications, University of Illinois at Urbana- \\ Champaign, 1205 W. Clark St., Urbana, IL 61801, USA \\ \{sclee,pbajcsy\}@ncsa.uiuc.edu
}

\begin{abstract}
This paper addresses the problem of intensity correction of fluorescent confocal laser scanning microscope (CLSM) images. CLSM images are frequently used in medical domain for obtaining 3D information about specimen structures by imaging a set of $2 \mathrm{D}$ cross sections and performing $3 \mathrm{D}$ volume reconstruction afterwards. However, the images of optical sections acquired from fluorescent CLSM demonstrate significant lateral intensity heterogeneity, which is well understood as one of the major barriers to accurate image analysis, e.g., quantitative analysis, segmentation, or visualization. The main contribution of the proposed work is in development of an intensity heterogeneity correction technique that (a) adjusts intensity heterogeneity in lateral plane of the sub-volume, (b) preserves fine structural details, and (c) enhances image contrast, by performing spatially adaptive mean-weight filtering. Furthermore, we provide data-driven parameter optimization method and evaluation metrics. The proposed filtering method is experimentally compared with several existing techniques by using four quality metrics, such as contrast, intensity heterogeneity (entropy) in low frequency domain, intensity distortion in high frequency domain, and saturation, with two realistic synthetic images and one CLSM image of a human histological section of uveal melanoma tissue.
\end{abstract}

\section{Introduction}

Visual inspection of medical specimens is one of the most common techniques in a medical domain used for diagnosis. Based on the need to investigate specimen characteristics at high spatial resolution, fluorescent Confocal Laser Scanning Microscopy (CLSM) imaging is frequently used for obtaining images of cross sections. Unfortunately, the intensity information often is distorted due to multiple specimen preparation and image acquisition limitations. Thus, there is a need to investigate techniques for fluorescent CLSM image intensity adjustment to support visual inspection tasks. Our work is primarily intended to support visual inspection of 3D volumes in virtual reality environments [1] to be quantitatively analyzed and correlated with information from other sensors. Nevertheless, it is well recognized that spatial intensity heterogeneity is a major barrier in acquiring reliable results during the above analyses [2],[3].

There are several known factors that cause spatial intensity heterogeneity, such as photo-bleaching, fluorescent attenuation along confocal (depth) axis, image acquisition factors [2],[4], variations of illumination exposure rate, spatially uneven distribu- 
tion of dye and the spatial characteristics of illumination beams [5], and fluorochrome micro-environment, e.g., $\mathrm{pH}$, temperature, embedding medium, etc [6]. However, it is infeasible to monitor all exact states of a fluorescent dye in an imaged specimen at a pixel resolution. Thus, it is very hard to develop an intensity heterogeneity correction method that would be directly linked to the sources of intensity heterogeneity coming from specimen preparation and image acquisition steps.

In past, intensity correction has been performed based on empirical correction methods for intensity loss [7], constant thresholding [8], iterative correction methods [9], 2D histogram [10], or estimations of intensity decay function [11]. However, most of these methods assume that the rate of photo-bleaching is spatially homogeneous in a lateral plane, can be characterized by an exponential function in depth [4], and mainly contributes to intensity loss along the specimen depth axis (z-axis) [12]. Nonetheless, these assumptions do not hold in a general case.

To solve the intensity correction problem in a lateral (x-y) plane, Histogram Equalization (HE) has been used in early applications [13], which leads to a uniform global intensity distribution in output image. However, it cannot effectively enhance local intensity variation due to its global property. To address this problem, Adaptive Histogram Equalization (AHE) has been used to adjust intensity variation locally by computing local histograms within spatially different windows [14]. A major problem of AHE is high sensitivity to noise, which results in amplification of undesired noise values. An improved approach to adjust local intensity variation is the Contrast Limiting Adaptive Histogram Equalization (CLAHE) [15]. It reduces noise amplification due to AHE by setting clipping limits and so removes boundary artifacts by background subtraction. Nevertheless, the main drawbacks of CLAHE are; (1) the parameters need to be manually selected. (2) There could be loss of fine details caused by intensity saturation. Alternatively, a model based approach is proposed by applying a bias field, e.g., intensity distortion map, to a polynomial function which is defined in prior [16]. However, this method requires prior knowledge about images, e.g., the degree of a polynomial function, and the computational complexity tends to increase exponentially upon the degree of a polynomial function.

In this work, we propose an intensity correction technique with data-driven parameter selection. The technique, we call mean-weight filtering, adjusts intensity heterogeneity in $\mathrm{x}-\mathrm{y}$ plane, preserves fine structural details, and enhances image contrast by performing spatially adaptive filtering, which is understood as highly salient in 3D visualization environment when examined by medical experts [1]. Although the intensity heterogeneity correction problem may be viewed as a restoration problem, we formulate the problem as an optimization problem since it is impossible to obtain the true uncorrupted intensity values for comparative purposes. Thus, we formulate the intensity heterogeneity correction problem as a search for an optimal, spatially adaptive, intensity transformation that maximizes intensity contrast with respect to background, minimizes overall spatial intensity variation for large area, e.g., low frequency domain, and minimizes distortion of intensity gradient for local features, e.g., high frequency domain, as shown in Fig. 1. We assume that the input image contains a single band (or a grayscale image) with spatially varying intensities. 

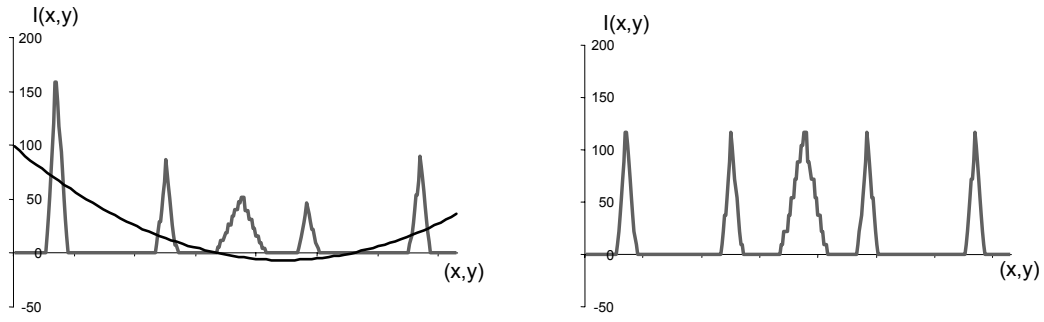

Fig. 1. Intensity Correction Problem: (left) measured intensity profile for CLSM images with intensity bias (solid curve) and (right) corrected intensity profile. Intensity bias has been corrected while preserving local intensity gradients.

\section{Mean Weight Filtering}

Our approach combines a kernel-based spatial filtering, and incorporates local and global image intensity analysis. The proposed intensity correction process consists of determining the background threshold intensity, optimizing the kernel size, constructing a set of $2 \mathrm{D}$ intensity correction maps for the volume, multiplying the intensity correction maps to the frames, and removing outlier pixels (speckle noise) from the corrected images based on statistical value ranges.

2D filter model: An image filtering approach to the presented intensity correction problem can be described as:

$$
g_{[a, b]}(x, y)=I(x, y) \cdot w_{[a, b]}(x, y)
$$

where $g_{[a, b]}(x, y)$ and $I(x, y)$ are the output and input pixel values at $(x, y)$ and $w[a, b](x, y)$ is the weighting coefficient computed over a pixel neighborhood $([x-a, x+a],[y-b, y+b])$. The spatial neighborhood $(2 a+1) \times(2 b+1)$, also denoted as a filtering kernel of size, is introduced to meet the requirement on local intensity gradient. Other requirements on intensity contrast and global spatial intensity variations are incorporated into the filter design by separating background according to a threshold $\delta$, and computing the weighting coefficient $w_{[a, b]}(x, y)$ as Eq. (2).

$$
w_{[a, b]}(x, y)=\left\{\begin{array}{cc}
\frac{\mu_{G}}{\mu_{L[a, b]}(x, y)} & \text { if } I(x, y)>\delta \text { and } \mu_{L[a, b]}(x, y) \neq 0 \\
1 & \text { otherwise }
\end{array}\right.
$$

where $\mu_{G}$ and $\mu_{L[a, b]}(x, y)$ are the global and local estimated sample means for foreground pixels only, and $\delta$ is a background threshold intensity value.

It is apparent that the weighting coefficients could be ill-defined when the local sample mean takes very small values (the spatial kernel belongs to background with some noise, $\mu_{L[a, b]}(x, y) \approx 0$.) To avoid this problem, input image is thresholded first, 
and then the values of global and local sample means are computed only over foreground pixels. In the filter design, we introduced two parameters, such as a background threshold $\delta$ and a kernel size $(2 a+1) \times(2 b+1)$.

Background separation: In general, the background threshold value could be determined by purely depending on images, such as variogram [17]. However, it is preferable that the noise model of different imaging techniques should be modeled differently based on known imaging physics such as Exponential, Rayleigh, Gaussian, Gamma, Poisson, or Weibull function [18]. In our background separation, we assume an exponential noise model for the CLSM background noise since it is well known that the noise in laser imaging can be modeled by an exponential function [13].

We derived $\delta$ by modeling a frequency function of the pixel intensities, i.e., a histogram, followed by fitting with underlying physical models of CLSM imaging. We model the frequency function of intensity values as a conjunction of background noise $\Lambda(v)$ in a photo-multiplier tube and foreground fluorescent pixel intensity distribution $\Phi(v)$, where $v$ is an intensity value. Assuming that $\Lambda(v)$ and $\Phi(v)$ follow an exponential and unknown exponential family of functional model respectively, we define the frequency functions as $\Lambda(v)=\alpha e^{\beta v}$ and $\Phi(v)=\gamma(v) e^{\kappa(v)}$ where $\alpha, \beta$ are constants and $\gamma(\cdot), \kappa(\cdot)$ are some functions for foreground intensity values. Since defined components are independent, assuming noise is additive, the frequency function $F(v)$ of output intensity can be modeled as a sum of $\Lambda(v)$ and $\Phi(v)$, which is $F(v)=\Phi(v)+\Lambda(v)$.

In particular, since the background pixels usually appear in low-intensity ranges and we only consider the left tail of the $F(v)$ where foreground intensity starts to contributes to $F(v)$ by fitting our noise model $\Lambda(v)$ to the low intensity range of $F(v)$. To compute the background frequency function, we estimated the parameters $\alpha, \beta$ using the least-squares methods [12] as following:

$$
\left[\begin{array}{cc}
\sum_{v=1}^{k} F(v) & \sum_{v=1}^{k} v F(v) \\
\sum_{v=1}^{k} v F(v) & \sum_{v=1}^{k} v^{2} F(v)
\end{array}\right]\left[\begin{array}{c}
\alpha \\
\ln (\beta)
\end{array}\right]=\left[\begin{array}{c}
\sum_{v=1}^{k} F(v) \ln (F(v)) \\
\sum_{v=1}^{k} v F(v) \ln (F(v))
\end{array}\right]
$$

where $F(v)$ is the frequency (e.g., pixel count) of the intensity value $v$ in the input image, $k$ is the bin number (intensity value) in $[1, n]$, and $n$ is the number of bins.

In order to find the threshold value which is dominantly determined by the background noise by photo-multiplier tube, we computed the sum of squared error $R(k)$ (Eq. (4)) by increasing $k$ from 3 to $n$ in Eq. (3), where $\Lambda_{k}(v)$ is an exponential function fit to first $k$ bins.

$$
R(k)=\left(\frac{1}{0.1 n} \sqrt{\sum_{v=1}^{0.1 n}\left(F(v)-\Lambda_{k}(v)\right)^{2}}\right)
$$

Since we approximated the foreground pixel intensity distribution for the left tail only, the sum of squared error is calculated for the pixel intensity values in $10 \%$ of 
low intensity value range, i.e., [1,25] in byte (8-bit) image. It approximates $\Lambda_{k}(v)$ in the input image well enough since $R$ is not much affected by large intensity values.

Finally, the background threshold value $\delta$ is determined by the value $k$ where the estimated function $\Lambda_{k}(v)$ best fits with the background noise in the input image such as $\delta=\arg \min (R(k))$.

Kernel size optimization: In order to compute local sample means $\mu_{L[a, b]}(x, y)$, one has to choose the size and shape of a filtering kernel for an input image. We first constrained the kernel shape to a rectangle. Then we selected a kernel size by maximizing the global contrast while minimizing the gradient distortion, e.g., high frequency distortion. We used a global contrast metric $\mathrm{C}$ described in [21] which incorporates one of the requirements of the intensity heterogeneity correction problem as follows.

$$
C=\sum_{i=1}^{m}\left\|f_{i}(I)-E(f(I))\right\| \times f(I)_{i}
$$

where $f(I)$ is the histogram (estimated probability density function) of all contrast values by using Sobel edge detector in an image $I, f_{i}(I)$ is the density of $i$-th bin, $E(f(I))$ is the sample mean of the histogram $f(I)$, and $m$ is the number of distinct contrast values in a discrete case. The equation includes the contrast magnitude term and the term with the likelihood of contrast occurrence. In general, image frames characterized by a large value of $C$ are more suitable for further processing than the frames with a small value of $C$.

To demonstrate the high frequency image difference between the original image and the processed image, we define a metric $D$ as follows:

$$
D=\sum_{v}^{M} \sum_{u}^{N}\left(I_{h f}^{\text {org }}(u, v)-I_{h f}^{a d j}(u, v)\right)^{2}
$$

where $I_{h f}^{\text {org }}$ and $I_{h f}^{a d j}$ are the high-pass filtered images of the original and the intensity adjusted image respectively. Finally, the filter size is selected by evaluating the maximum value of the ratio $C / D$ denoted as a measure of image saliency.

Speckle noise removal: One of the side products of the mean-weight filtering is an easy detection of speckle noise in the background. Speckle noise is characterized by a pixel with very few or no neighboring pixels and the mean-weight filtering generates very high intensity correction value for the speckle pixels. We have eliminated speckle noise by removing the pixels with abnormally high intensity correction values statistically, and accepted the pixels with values within the range $[0, \mu+4 \sigma](99.99 \%$ of pixels are included), where $\mu$ and $\sigma$ are the sample mean and standard deviation of the intensities in the corrected image. Finally, the values within the range $[0, \mu+4 \sigma]$ are normalized to $[0,255]$ to meet the dynamic range of output images $(8$ bits per pixel). 


\section{Experimental Results}

Simulation Results: Fig. 2 (left) shows a bias-free synthetic image (shown as horizontal and vertical bars with different thickness and spacing, and Fig. 2 (middle) and (right) show intensity distorted images by pre-defined intensity variations (bias fields). Background noise is simulated by adding random exponential synthetic noise with density function $\Lambda(v)=0.3 e^{0.3 v}$.

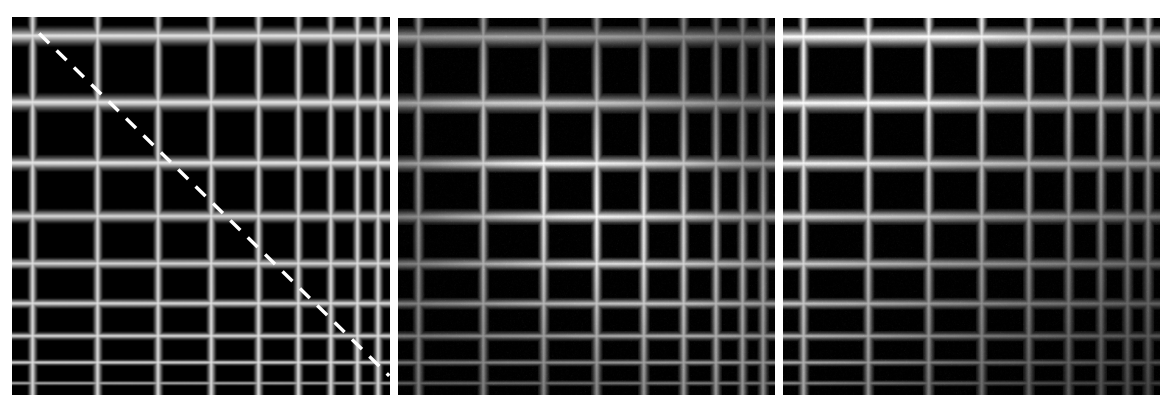

Fig. 2. (left) bias-free image (dotted line is shown to illustrate the intensity profile later in Fig. 7), (middle, right) images with intensity distortion with synthetic noise.

Fig. 3. (left) shows the curves of residuals $R(\cdot)$ for the two simulation images in Fig. 2. (middle, right) (see Eq. (4)). The background thresholds are selected based on the minimum value of the curves except the low value range between 1 and 3 , which were 20 for both simulation images. Next, to optimize the kernel size for each image, we calculated the image saliency $(C / D)$ for kernel sizes from 3 to 51 , shown in Fig. 3. (right). Generally, it is observed that; (1) for small kernels, the contrast $C$ is maximized but the high frequency distortion is large; (2) for large kernels, the high frequency distortion is minimized but the contrast $C$ is compromised. Therefore, the simulation results verified that the optimal kernel size is achieved by maximizing the contrast divided by the high frequency difference $(C / D)$. In our simulation experiments, we calculated $C / D$ using kernel sizes from 3 to 51 pixels wide, and obtained
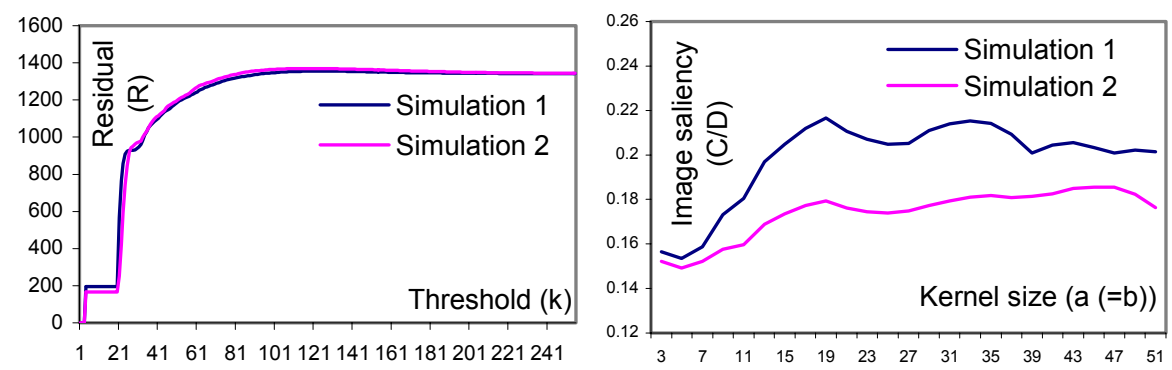

Fig. 3. (left) Residual for background thresholding in Eq, (4), and (right) image saliency as a function of the kernel size (see Eq. (5) and Eq. (6)). 

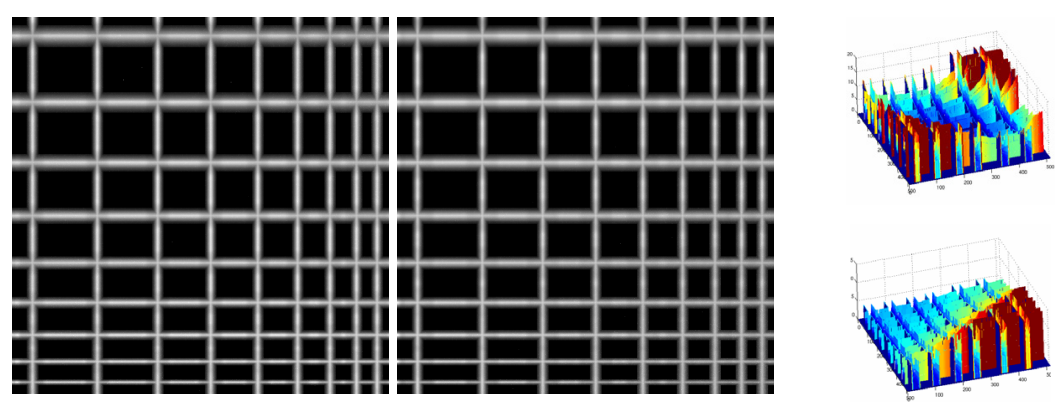

Fig. 4. (left, middle) Intensity corrected images using the mean-weight filtering with the kernel size of 33 (left) and 47 (middle) respectively. (right) Intensity correction maps (pseudo colored) where upper and lower map corresponds to the (left) and (middle) image respectively.

the optimum values equal to 33 and 47 pixel widths respectively (see the peaks in Fig.3 (right)).

Given the background threshold and kernel size in Fig. 3, we show the intensity corrected images by the mean weight filtering in Fig. 4. (left and middle). Fig. 4. (right) shows the intensity correction maps applied to the intensity distorted (uncorrected) images. The intensity correction maps demonstrate higher weight in dark local regions than in bright local regions. Regardless of the type of a bias field, all intensity corrected images show noticeable improvement such that the mean weight filtering corrects intensity heterogeneity over spatially large area while preserving the edge gradients (minimum high frequency distortion) over spatially small area.

For visual comparison, we show the intensity correction results for the simulation images using Histogram equalization (HE), Histogram equalization with background separation (HEB) (background threshold $=20$ ), and CLAHE (see Fig. 5). One could notice that HE saturated most of pixels, amplified background noise, and removed edge gradient significantly. HEB separated out background noise, but the edge gradient is not preserved well similarly to the HE, and CLAHE relatively well preserved the edge gradient, but the spatial (x-y) intensity heterogeneity has not been corrected in comparison to the Mean weight filtering.

To demonstrate the spatial intensity correction result, Fig. 6. shows the intensity profiles (along the dotted line Fig. 2. (left) for the simulation 2 (see Fig. 2 (right)) of the uncorrected, HE, HEB, CLAHE, and the mean weight filtering. The intensity profile clearly demonstrates that the intensity along $x-y$ plane is best corrected by the mean weight filtering while preserving local intensity gradients, i.e., the peaks of intensity value remains between 190 and 200 . 

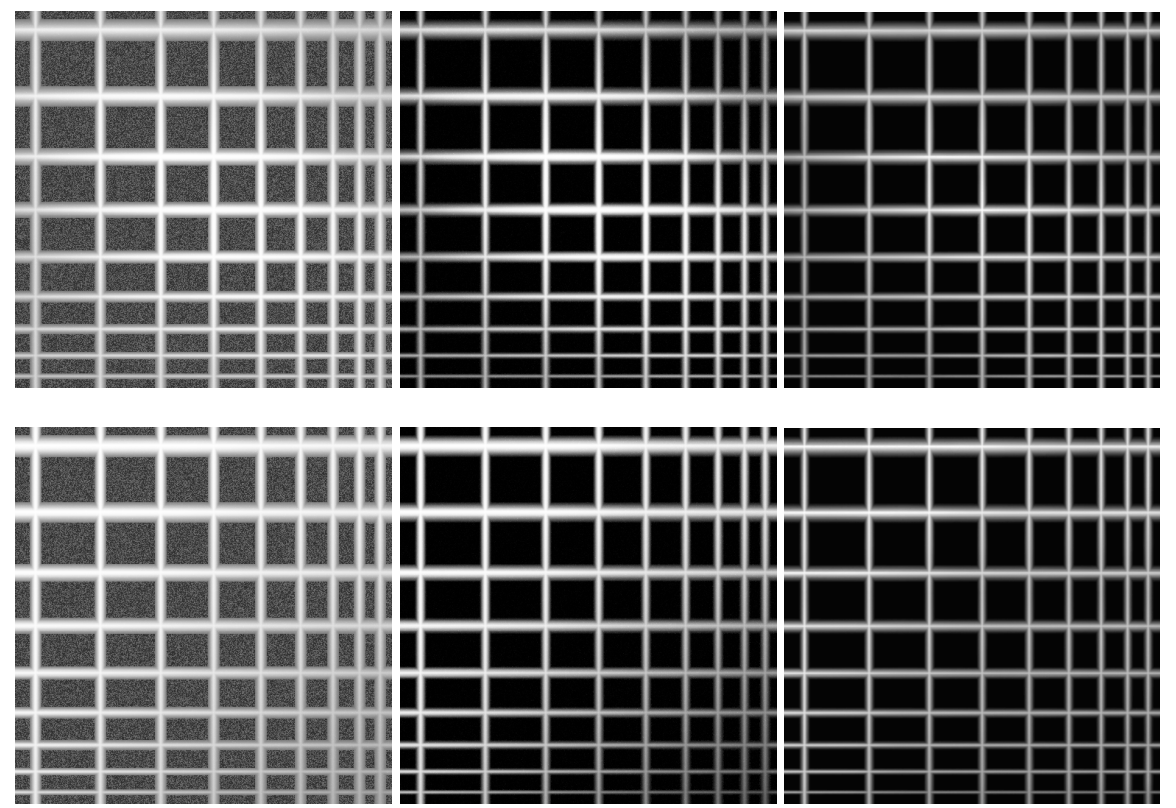

Fig. 5. Result of Histogram Equalization, Histogram Equalization with background thresholding, and CLAHE for simulation 1 (upper three images) and simulation 2 (lower three images).

Comparative Results for CLSM Images: We applied the mean weight filtering method to a real CLSM image. The image was acquired with a Leica SP2 laser scanning confocal microscope (Leica, Heidelberg, Germany) using the 40X objective with $605 \sim 700 \mathrm{~nm}$ excitation wavelength range for the test specimens. The image was stored in tagged information file format (TIFF) with 512 by 512 pixel resolution.
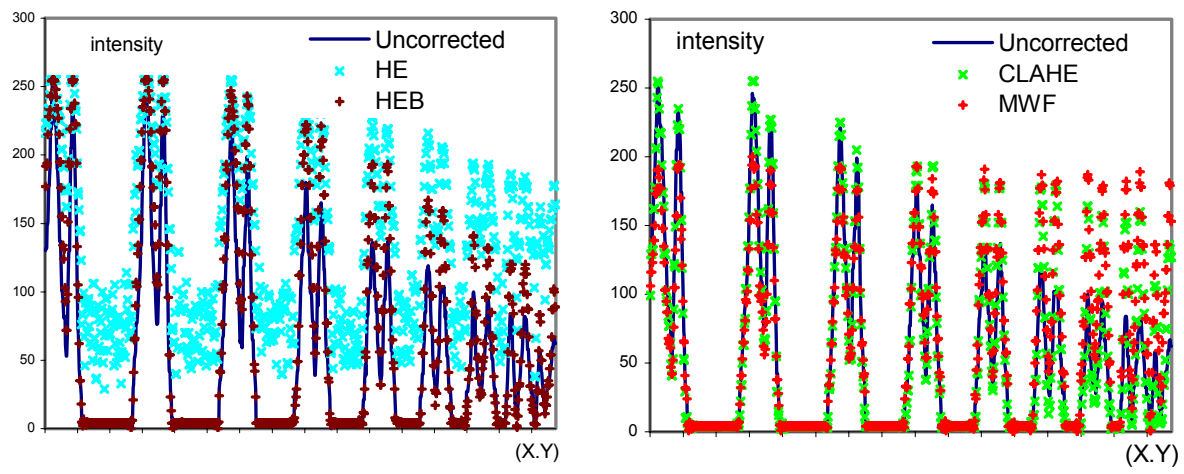

Fig. 6. Intensity profile along the red dotted line in Fig. 3 (left) for the simulation image 2: Intensity profiles (left) for uncorrected, HE, and HEB, and (right) for uncorrected, CLAHE, and mean-weight filtering. 

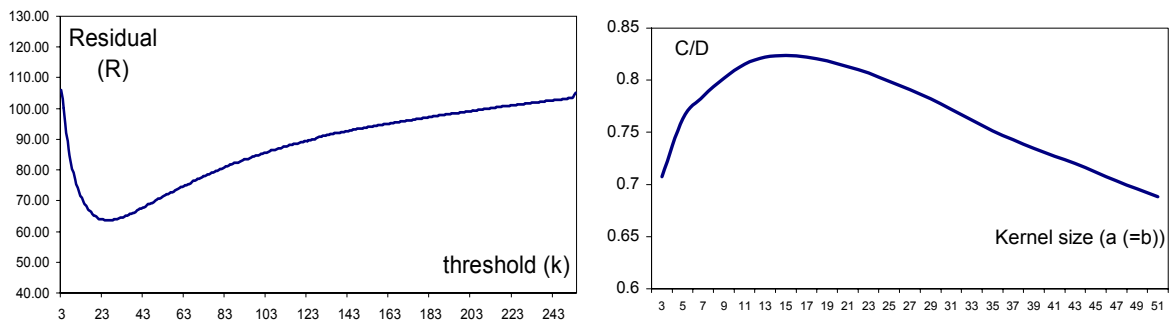

Fig. 7. Residual of the estimated function for the input image in Fig. 8. The intensity threshold minimizing $\mathrm{R}$ is 26 (left), and the optimal kernel size (maximizing C/D) is 15 (right).

First, we decided the background threshold and the kernel size by the proposed method in the Section 2, which correspond to $\delta=26$ and $a=b=15$ (see Fig. 7.)

In order to quantitatively asses the quality of multiple filtering techniques for real CLSM images, multiple intensity correction results are compared by image contrast $C$ (Eq. (5)), high frequency distortion $D$ (Eq. (6)), the low frequency intensity heterogeneity $S$ (evaluated by the image entropy in low frequency domain [20]), and the number of saturated pixels $N$ as defined in Eq. (7).

$$
N=\sum_{v}^{M} \sum_{u}^{N} \operatorname{sat}\left(I^{\text {org }}(u, v), I^{\text {adj }}(u, v)\right)
$$

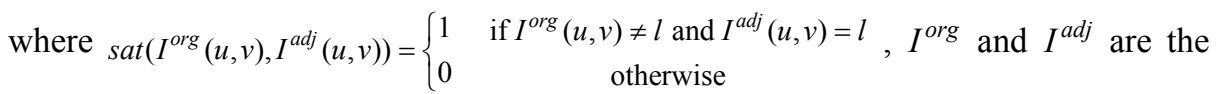
original and intensity adjusted images, and $l$ is the maximum intensity value of the original image, e.g. 255 in a byte image. For the best image quality, it is desirable to achieve large $C$ and small $S, D$, and $N$.

Fig. 8. shows the input CLSM image with the processed results by existing and the mean-weight filtering method. One could visually notice that the histogram equalization method removes best intensity heterogeneity in large spatial regions but maintains low visual saliency and, most importantly, removes edge details (high frequency distortion) (see the second image in Fig. 9.). The histogram equalization with background threshold $(\delta=26)$ and Contrast Limited Adaptive Histogram Equalization demonstrate similar removal of edge gradient and edge details. To demonstrate the effect of edge gradient, we show a sub-region of the intensity corrected images in Fig. 9. They show that most of pixels around the edge were saturated using HE, HEB, or CLAHE while the mean weight filtering well preserves edge gradient.

In addition to visual assessments, we show the quantitative evaluation of the compared techniques with four quality metrics in Table 1. In general, an optimal intensity correction technique has to meet multiple optimization criteria, for example, minimize $S, D$ and $N$, and maximize $C$. For both measured test images, Table 1 shows that the mean-weight filtering achieves a normalized metric that is about 1.5 to 1.7 times larger than the second best performing technique. 


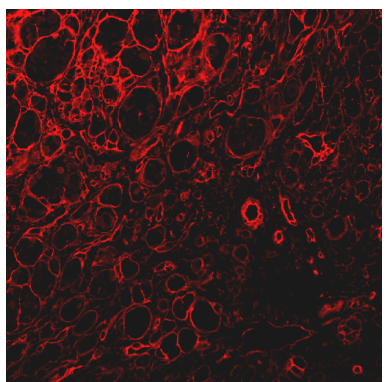

(a)

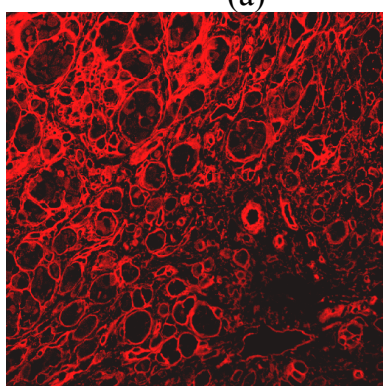

(d)

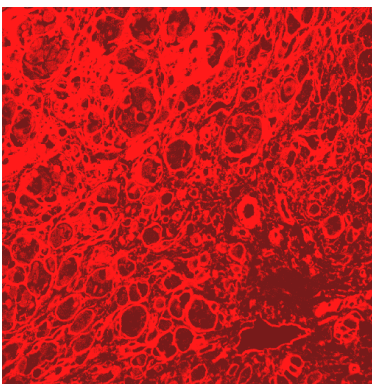

(b)

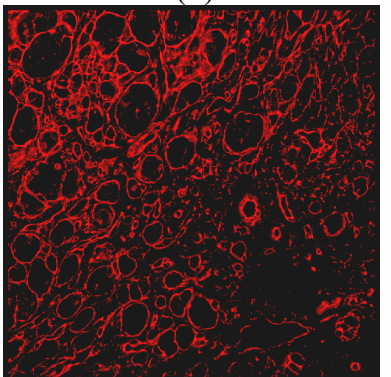

(e)

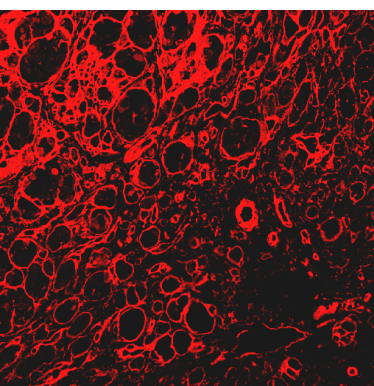

(c)

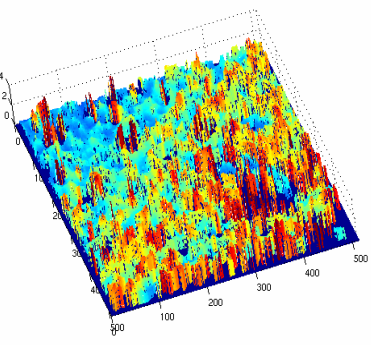

(f)

Fig. 8. CLSM test image: (a) Original test image, (b) histogram equalization, (c) histogram equalization with background thresholding, (d) CLAHE ("adapthisteq()" with default setting by Matlab Image Processing Toolbox, Build. R12), (e) mean weight filtering, and (f) intensity correction maps by the mean weight filtering.
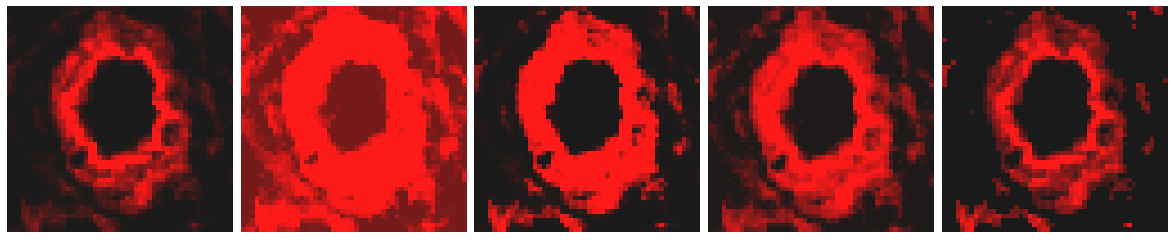

Fig. 9. Sub-region of intensity corrected images in Fig. 8. (from left, original image, HE, HEB, CLAHE, and Mean-weight filtering)

Table 1. Evaluation results in Fig. 8. The first and second best results are shown in bold and italic respectively.

\begin{tabular}{ccccc}
\hline CLSM Image & HE & HEB (26) & CLAHE & MWF (26,15) \\
\hline C & 17.15 & $\mathbf{1 7 . 3 4}$ & 17.20 & 16.26 \\
S & 74.27 & 73.40 & 74.86 & $\mathbf{6 6 . 2 4}$ \\
D & 29.88 & 23.93 & 28.02 & $\mathbf{2 1 . 9 8}$ \\
N & 330 & 330 & 334 & $\mathbf{0}$ \\
\hline
\end{tabular}




\section{Conclusion and Discussion}

We introduced an intensity heterogeneity correction technique that adjusts intensity heterogeneity of 2D images, preserves fine structural details, and enhances the image contrast by performing spatially adaptive mean-weight filtering. The proposed technique was designed by formulating the problem requirements, defining image quality metrics, and then optimizing filter parameters based on an image analysis. We conclude that the developed mean-weight filtering technique outperforms other intensity correction methods by at least a factor of 1.5 when applied to fluorescent CLSM images.

Although automatic selection of a kernel size shows optimized the global image saliency, it is worth to consider some images with a mixture of different structures (e.g., edge thickness) that require multiple kernel sizes per image for different regions of interest. If a user chooses to select a kernel size on his own, we would provide the following considerations: (a) A large kernel tends to preserve the detail of rather large area, e.g., thick edge or spatial intensity heterogeneity in a feature region, and extremely large kernels correct minimally intensities in x-y plane. (b) A small kernel generates visually salient images by highlighting sharp intensity changes, e.g., small intensity discontinuities. However, extremely small kernels correct high frequency intensity change which is typically considered as edge gradient (and need to be preserved). (c) A kernel size could be selected based on edge thickness: for thin edges, a smaller kernel size is preferred since only high frequency component should be corrected. For thick edges, a larger kernel should be used since a low frequency component should be corrected while preserving a high frequency component. Fig. 10 shows the mean weight filtering results with different kernel sizes.
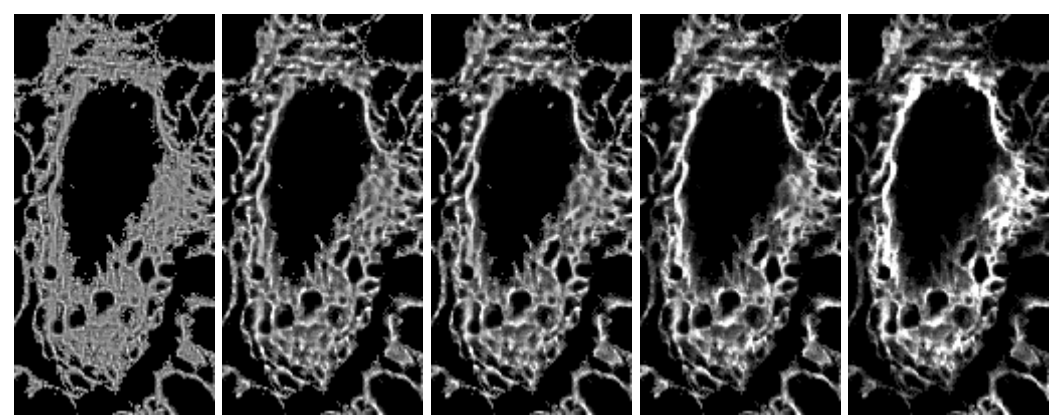

Fig. 10. Mean weight filtering with different kernel sizes: From left, the kernel width is equals to $3,7,9,21,51$.

\section{Reference}

1. Chen, X., Ai, Z., Rasmussen, M., Bajcsy, P., Auvil, L., Welge, M., Leach, L., Folberg, R.: Three-dimensional reconstruction of extravascular matrix patterns and blood vessels in 
human uveal melanoma tissue: Preliminary findings. Invest. Ophthal. \& Vis. Sci., 44 (2003) 2834-2840

2. Benson, D., Bryan, J., Plant, A., Gotto, A., Smith, L.: Digital Imaging fluorescence microscopy: Spatial heterogeneity of photobleaching rate constants in individual cells. J. Cell Biol. 100 (1985) 1309-1323

3. Jungke, M., Seelen, von W., Bielke, G. , Meindl, S. et al.: A system for the diagnostic use of tissue characterizing parameters in NMR-tomography. Proc. of Info. Proc. in Med. Imaging, IPMI'87, 39 (1987) 471-481

4. Rigaut, J., Vassy, J.: High-resolution 3D images from confocal scanning laser microscopy: quantitative study and mathematical correction of the effects from bleaching and fluorescence attenuation in depth. Anal. Quant. Cytol. 13 (1991) 223-232

5. Oostveldt, P. V., Verhaegen, F., Messen, K.: Heterogenous photobleaching in confocal microscopy caused by differences in refractive index and excitation mode. Cytometry 32 (1998) 137-146

6. Tauer, U., Hils, O.: Confocal Spectrophotometry, in Sci. and Tech. Info., Sp. issue :Confocal Microscopy, CDR 4 (2000) 15-27

7. Rodenacker, K., Aubele, P., Hutzler, M., Adiga, P.: Groping for quantitative digital 3-D image analysis: an approach to quantitative fluorescence in situ hybridization in thick tissue sections of prostate carcinoma. Anal. Cell. Pathol. 15 (1997) 19-29

8. Irinopoulo, T., Vassy, J., Beil, M., Nicolopoulo, P., Encaoua, D., Rigaut, J.: 3-D DNA image cytometry by confocal scanning lasermicroscopy in thick tissue blocks of prostatic lesions. Cytometry, 27 (1997) 99-105

9. Roerdink, J., Bakker, M.: An FFT-based method for attenuation correction in fluorescence confocal microscopy. J. Microsc. 169 (1993) 3-14

10. Liljeborg, A., Czader, M., Porwit, A.: A method to compensate for light attenuation with depth in 3D DNA image cytometry using a confocal scanning laser microscope. J. Microsc. 177 (1995) 108-114

11. Kervrann, C., Legland, D., Pardini, L.: Robust incremental compensation of the light attenuation with depth in 3D fluorescence microscopy. J. Microsc., 214 (2004) 297-314

12. Oostveldt, P., Verhaegen, F., Messens, K.: Heterogeneous photobleaching in confocal microscopy caused by differences in refractive index and excitation mode. Cytometry, 32 (1998) 137-146

13. Gonzalez, R., Woods, E.: Digital Image Processing. 2nd ed., Prentice hall, (2002)

14. Pizer, S. M., Zimmerman, J. B., Stabb, E.: Adaptive grey level assignment in CT scan display. J. Comp. Assist. Tomography, 8 (1984) 300-305

15. Pisano, E., Zong, S., Hemminger, M., De Luca, M., Johnsoton, R., Muller, K., Braeuning, M., Pizer, S.: Contrast Limited Adaptive Histogram Equalization Image Processing to Improve the Detection of Simulated Spiculations in Dense Mammograms. J. Digital Imaging, 11(4) (1998) 193-200

16. Styner M, Brechbuhler C, Szekely G., Gerig G.: Parametric estimate of intensity inhomogeneities applied to MRI. IEEE Trans Med Imaging, 19(3) (2000) 153-65

17. Sanchez-Brea, L.M., Bernabeu, E.: On the standard deviation in CCD cameras: a variogram-based technique for non-uniform images. J. Electronic Imaging 11(2) (2002) 121-126.

18. Hu, J., Razdan, A., Nielson, G., Farin, G., Baluch, D., Capco, D.: Volumetric Segmentation Using Weibull E-SD Fields, IEEE Trans. on Vis. and Comp. Graphics, 9(3) (2003)

19. Weisstein, E. Least Squares Fitting--Exponential. from MathWorld--A Wolfram Web Resource. http://mathworld.wolfram.com/LeastSquaresFittingExponential.html

20. Mangin, J.: Entropy minimization for automatic correction of intensity nonuniformity Math. Method in Biomed. Image Analysis (MMBIA), (2000) 162-169

21. Bajcsy P., Groves, P.: Methodology for Hyperspectral Band Selection, Photo. Eng. and Remote Sensing J., 70 (2004) 793-802 